DRAFT VERSION JULY 3, 2018

Preprint typeset using LTEX style emulateapj v. 5/2/11

\title{
SHADOWS CAST BY A WARP IN THE HD 142527 PROTOPLANETARY DISK
}

\author{
S. MARINO ${ }^{1,2}$, S. PEREZ ${ }^{1,2}$ AND S. CASASSUS ${ }^{1,2}$ \\ ${ }^{1}$ Departamento de Astronomía, Universidad de Chile, Casilla 36-D, Santiago, Chile and \\ ${ }^{2}$ Millenium Nucleus "Protoplanetary Disks in ALMA Early Science," Universidad de Chile, Casilla 36-D, Santiago, Chile \\ Draft version July 3, 2018
}

\begin{abstract}
Detailed observations of gaps in protoplanetary disks have revealed structures that drive current research on circumstellar disks. One such feature is the two intensity nulls seen along the outer disk of the HD 142527 system, which are particularly well traced in polarized differential imaging. Here we propose that these are shadows cast by the inner disk. The inner and outer disk are thick, in terms of the unit-opacity surface in $H$ band, so that the shape and orientation of the shadows inform on the three-dimensional structure of the system. Radiative transfer predictions on a parametric disk model allow us to conclude that the relative inclination between the inner and outer disks is $70 \pm 5 \mathrm{deg}$. This finding taps the potential of high-contrast imaging of circumstellar disks, and bears consequences on the gas dynamics of gapped disks, as well as on the physical conditions in the shadowed regions.
\end{abstract}

Subject headings: protoplanetary disks: general stars: individual(HD 142527)

\section{INTRODUCTION}

The study of planet formation is rapidly being revolutionized by observational breakthroughs, such as the direct images of protoplanetary gaps in near-infrared scattered light. A particularly interesting target is the Herbig Ae/Be star HD 142527, at a distance of $\sim 140$ pc. The disk surrounding HD 142527 boasts the largest inner cavity known, with a $1^{\prime \prime}$ radius and seen at a close to face-on orientation, with an inclination of about 24 deg (Fukagawa et al. 2006, Fujiwara et al. 2006). Improvements in $K_{\mathrm{s}}$-band coronagraphy revealed fine structure in the outer disk, including two intriguing intensity nulls (Casassus et al. 2012) that break the continuity of the outer disk. In this Letter we propose that these are shadows cast by a warped inner disk.

Recent polarized differential imaging (PDI) of HD 142527 at $H$ - and $K_{\mathrm{s}}$-bands revealed a striking view of its dustdepleted gap, outer disk and spiral structures (Canovas et al. 2013, Avenhaus et al. 2014; Rodigas et al. 2014). These $H$ band polarized intensity images outline the outer-disk nulls with the best available detail thus far. One null is found $\sim 11.5 \mathrm{~h}$ (North of the star), and the other is found at $7 \mathrm{~h}$ (South). The Northern null is particularly puzzling as it coincides with the location of the dust density peak, whose spatial distribution is shaped into a large crescent (Ohashi 2008 . Casassus et al.|2013b; Fukagawa et al.(2013). Thus the Northern null cannot be interpreted as a lack of material.

As witnessed by a bright thermal IR central point source, well in excess of the photospheric emission, the large dustdepleted cavity of the HD 142527 disk is in fact a gap (Fujiwara et al. 2006; Verhoeff et al. 2011; Rameau et al. 2012). While knowledge of the outer edge of the gap is limited by theory rather than observational detail, the inner regions of the gap are poorly resolved. $\mathrm{A} \sim 10^{-7} M_{\odot} \mathrm{yr}^{-1}$ stellar accretion rate (Garcia Lopez et al. 2006) would quickly deplete the inner disk, whose dust mass is estimated at $\sim 10^{-9} M_{\odot}$ (Verhoeff et al. 2011). This inner disk can be thought of as a transient feature of stellar accretion: it is the convergence point of matter being accreted from the mass reservoir in the outer disk (Casassus et al. 2013b a; Casassus 2015a). Long-

smarino@das.uchile.cl baseline optical interferometry (VLTI) has resolved this inner disk, and found it to be highly crystalline (van Boekel et al. 2004) - but its orientation and extent remain elusive.

Motivated by our research on the intra-cavity gas kinematics (Casassus et al.2013a; Casassus 2015a) we considered the possibility of a continuous warp linking different orientations between the outer and inner disks. The underlying parametric disk model is documented in Section 2, along with the radiative transfer setup we implemented to calculate the emergent specific intensity fields. Our results are presented in Sec. 3 While the inner disk intercepts stellar light, shadows are cast on the outer disk such that their shape and orientation are sensitive measures of the inner disk orientation. We briefly consider the consequences of this finding in Sec. 4

\section{PARAMETRIC MODELING}

\subsection{Underlying physical structure}

We constructed a synthetic model of HD 142527 inspired by multiwavelength observations and gas kinematics, and detailed enough to provide a comparison point with the near-IR scattered light images as well as the radio continuum emission and gas kinematics. As explained in a companion paper (Casassus 2015b), this synthetic model matches the observed spectral energy distribution (SED). Here we document the aspects of the model that are relevant to this report on the $H$ band images.

The model consists of a star at the center of a dusty disk separated in 3 zones with different density structures, dust grain size distributions and compositions: an inner disk, a gap and an outer disk. A schematic render of the model is provided in Figure 1. We model the star using a Kurucz template spectrum (Castelli \& Kurucz 2003) at a temperature of $7750 \mathrm{~K}$ and with a radius of $3.3 R_{\odot}$, to be consistent with the extinction of $A_{R}=1.3 \pm 0.3$ reported in Close et al. (2014).

The inner disk starts at 0.3 au and cuts off at $10.0 \mathrm{au}$. It is described by a surface density proportional to $r^{-1}$ and a scale height of $1.0 \mathrm{au}$ at $10 \mathrm{au}$ with a flaring exponent of 1.1 . It is composed of amorphous carbon and silicate grains with sizes between 0.1 to $2.5 \mu \mathrm{m}$, with a total dust mass of $5.0 \times 10^{-9}$ $\mathrm{M}_{\odot}$. We introduce an inclination of $\alpha=70^{\circ}$ to this region with respect to the outer disk midplane. 
The gap spans 120 au from 10.0 au. It is described by a surface density proportional to $r^{-1}$ and a scale height of 18 au at 100 au with a flaring exponent of 1.6. It is composed of amorphous carbon and silicate grains with sizes between 1.0 to $10.0 \mu \mathrm{m}$. The total dust mass of this section is $1.0 \times 10^{-8}$ $\mathrm{M}_{\odot}$. The disk in this section connects the inner and outer regions varying the inclination linearly from $70^{\circ}$ to $0^{\circ}$ between 10 and $15 \mathrm{au}$, where it matches the outer disk orientation. A larger warp would have been obvious in the ${ }^{12} \mathrm{CO}$ kinematics inside the gap (Perez et al. 2014), with a concomitantly larger region where the inclination crosses the plane of the sky.

Finally, the outer disk extends over 115 au to 300 au with a rounded disk wall between 115 to $140 \mathrm{au}$. It is composed of $3.0 \times 10^{-6} \mathrm{M}_{\odot}$ of amorphous carbon grains with sizes ranging from 1 to $10 \mu \mathrm{m}$ and $1.0 \times 10^{-2} \mathrm{M}_{\odot}$ of silicate grains with sizes ranging from $100 \mu \mathrm{m}$ to $5 \mathrm{~cm}$. The dust masses inferred in our models are biased by the lack of grain porosity. The resulting dust masses are also directly affected by uncertainties on the internal densities.

We assume that the small grains that account for the bulk of the near-IR opacity approximately follow the gas background, and we define an axisymmetric gas distribution with a rounded disk wall (Mulders et al. 2013; Lubow \& D'Angelo 2006) described by the following surface density:

$$
\begin{aligned}
& \bar{\Sigma}_{g}\left(r<r_{c}\right)=\Sigma_{c}\left(\frac{r}{r_{c}}\right)^{-\gamma} \exp \left[-\left(\frac{1-r / r_{c}}{w}\right)^{3}\right], \\
& \bar{\Sigma}_{g}\left(r \geq r_{c}\right)=\Sigma_{c}\left(\frac{r}{r_{c}}\right)^{-\gamma},
\end{aligned}
$$

where $\gamma=6, w=0.1$ and $r_{c}=148.0$ au. Then we modulate this distribution to create a maximum gas pressure in azimuth, which is described by the following equations:

$$
\begin{aligned}
\Sigma_{g}(r, \phi) & =\bar{\Sigma}_{g}(r)\left[1+A(r) \sin \left(\phi+\frac{\pi}{2}\right)\right], \\
A(r) & =\frac{c-1}{c+1} \exp \left[-\frac{\left(r-R_{s}\right)^{2}}{2 H^{2}}\right],
\end{aligned}
$$

with $R_{s}=148$ au and where the azimuthal contrast in surface density is set to $c=10.0$. The volume density follows with a standard vertical Gaussian distribution:

$$
\rho_{g}(r, z, \phi)=\frac{\Sigma_{g}(r, \phi)}{\sqrt{2 \pi} H} \exp \left[-\frac{z^{2}}{2 H^{2}}\right],
$$

with $H(r)=20.0(r /(130 \mathrm{au}))^{1.17}$. The exact value of this flaring exponent is not well constrained.

This parametric model also includes the effect of dust trapping, following the procedure described in Birnstiel et al. (2013) and Pinilla et al. (2012). However, the bulk of the opacity in $H$-band is driven by particles well below the sizes required for efficient aerodynamic coupling, and so the effects of dust trapping in the outer disk are not relevant to this report. The runs detailed in Sec. 3 confirm that the outer disk is optically thick at $\mathrm{H}$-band and that the scattered light does not trace the crescent shape seen in the submm.

\subsubsection{Emergent intensities}

We use RADMC $3 \mathrm{D}^{1}$ for radiative transfer computations (version 0.38, Dullemond et al. 2014). Scattering and polarization for last scattering are treated with scattering matrices

\footnotetext{
${ }^{1}$ http://www.ita.uni-heidelberg.de/ dullemond/software/radmc-3d/
}

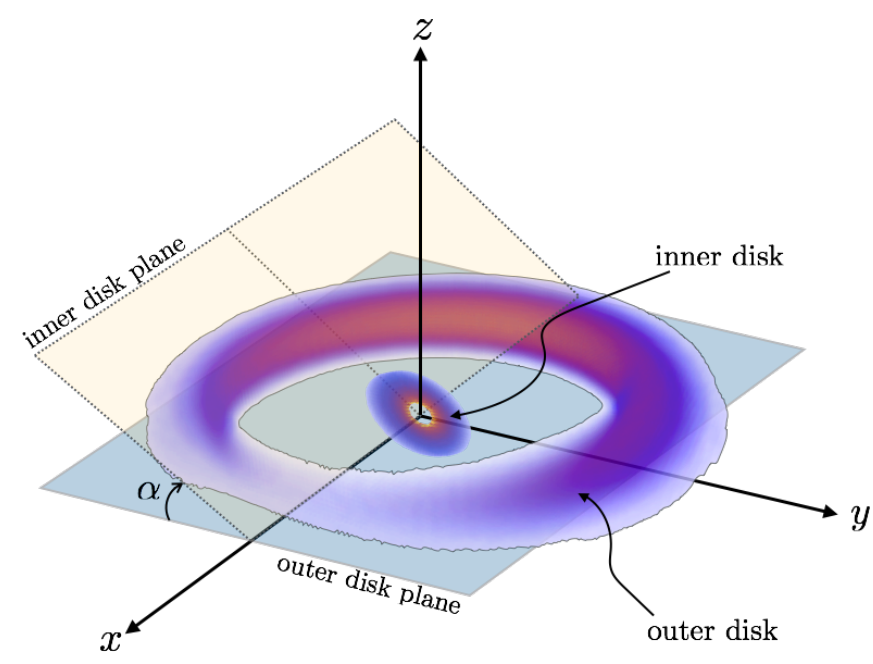

FIG. 1.- Schematic view with arbitrary orientation of the parametric model presented in Sec. 2 The central star is placed at the origin. The outer disk lies in the $x-y$ plane. The angle $\alpha$ is the relative inclination between the midplane of the outer disk and the plane of the inner disk. The dust mass density distribution of the inner disk and outer disk sections are rendered in false color. The gap is shown devoid of material for simplicity. The inner disk is scaled up in size and density for better visualization.

for our different dust species, each one with a power law distribution in grain sizes with exponent -3.5 . To compute the full dust opacity and scattering matrices we made use of complementary codes in RADMC3D and a code from Bohren \& Huffman (1983) for "Mie solutions" to scattering by homogeneous spheres. We used the optical constant tables for amorphous carbon grains from $\mathrm{Li} \&$ Greenberg (1997), and for silicate grains we used Henning \& Mutschke (1997).

We implemented our model in RADMC3D using spherical coordinates, with regular spacing for the azimuthal angle, and logarithmic spacing in radius and colatitude (polar coordinate). Thus, the grid is naturally refined near the inner disk and the midplane. The radial grid is additionally refined as it approaches the inner wall of the outer disk (near $140 \mathrm{au}$ ) to ensure a gradual transition from the optically thin gap to the optically thick outer disk. We used $10^{6}$ cells in total, half of them covering the inner disk and gap, and the rest sampling the outer disk. The number of points in the radial, azimuthal and polar grid meshes is 100 each.

As proposed by Fujiwara et al. (2006), the Eastern side is probably the far side since it is broader and brighter in the thermal IR. This orientation also implies that the observed IR spiral pattern is consistently trailing (Fukagawa et al. 2006, Casassus et al. 2012; Canovas et al. 2013; Avenhaus et al. 2014), even in their molecular-line extensions into the outer disk (Christiaens et al. 2014). Hence we calculated the synthetic $H$-band images by inclining the system at $24 \mathrm{deg}$ with respect to the plane of the sky ${ }^{2}$, along a position angle (PA) of $-20 \mathrm{deg}$.

\section{RESULTS}

In order to constrain the PA of the inner disk and $\alpha$, its inclination with respect to the outer disk, we studied different orientations while trying to reproduce the shape and position of the nulls seen in scattered and polarized light. In Fig. 2 we summarize the radiative transfer predictions of 5 different configurations. PAs much different from -8 are ruled out, as

\footnotetext{
2 the outer disk defines the plane of the system
} 


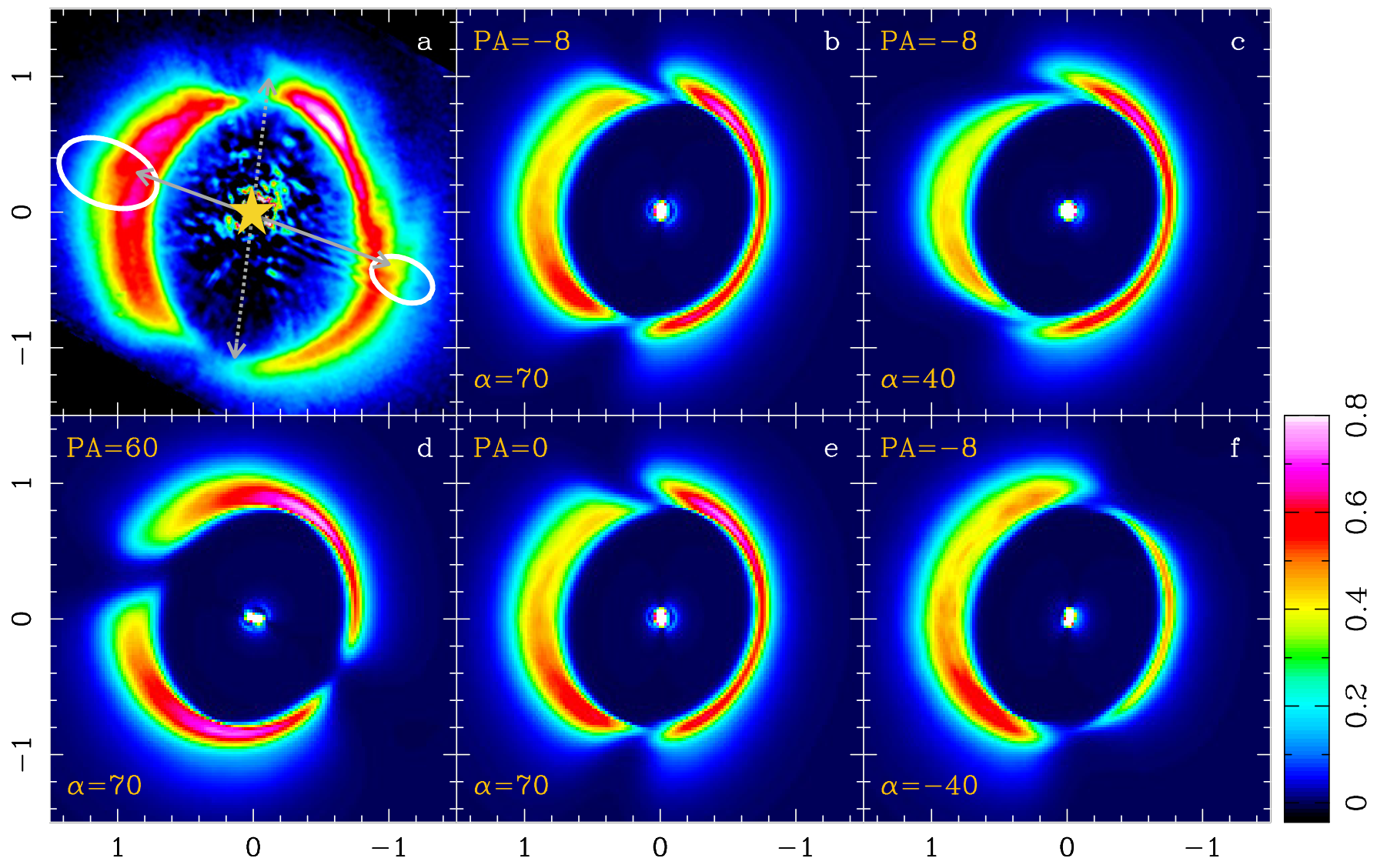

FIG. 2.- Impact of the inner disk orientation on the H-band light scattered off the outer disk. a: NACO-PDI H-band image from Avenhaus et al. (2014), compared with the $\mathrm{C}^{18} \mathrm{O}(2-1)$ emission at systemic velocity from Perez et al. (2014). The $\mathrm{C}^{18} \mathrm{O}(2-1)$ emission, represented here as one white contour at 0.75 maximum, shows that the position angle (PA) of the outer disk is at -20 deg East of North, and perpendicular to the solid gray double-arrow, while the position angle of the intensity nulls is indicated by the dashed double-arrow ( $-8 \mathrm{deg})$. b-f: Radiative transfer prediction for polarized intensity in $\mathrm{H}$-band, for different inner disk PAs (indicated in degrees on the plots), and for different relative inclinations $\alpha$ between the inner and the outer disks, such that the net inclination of the inner disk is $(-20+\alpha)$ deg relative to the plane of the sky. The $x-$ and $y-$ axis indicate offset along RA and DEC, in arcsec.

even a PA of 0 (see Fig. 2e) displaces the southern shadow so that it is inconsistent with the observations. In parallel, for low and negative $\alpha$, the inclination and orientation of the shadows do not fit the shape of the nulls (see Fig. 25\&f). A qualitative match with the observations is obtained with an inner disk inclined at $\alpha=70 \mathrm{deg}$ relative to the outer disk, and along a PA of $-8 \mathrm{deg}$ (Fig. 2p). We can rule out configurations with PAs beyond $10 \mathrm{deg}$ of $-8 \mathrm{deg}$, so that the $1 \sigma$ error bar is about 5 deg. Likewise, the relative inclination is constrained within 60 to $80 \mathrm{deg}$, so that the $1 \sigma$ error is also $\sim 5 \mathrm{deg}$.

An inner disk orientation along a PA of $\sim 60^{\circ}$ has been proposed by Pontoppidan et al. (2011), based on long-slit spectroscopy of the CO $4.67 \mu \mathrm{m}$ line, along with a purely Keplerian disk model. However, as illustrated in Fig. 2d, such an orientation can be discarded from the $H$-band imaging. It is possible that non-Keplerian kinematics may have biased the orientation inferred from the ro-vibrational $\mathrm{CO}$.

It is interesting that our models predict a peak $H$-band intensity at the same position as in the observations, at $\sim 1.5 \mathrm{~h}$ (North-North-West). However, the second peak in the PDI image to the North-East does not coincide with our radiative transfer predictions. This can be due to an effect of fine structure in the outer disk, or to deviations from a perfect ring, or to the stellar offset from the center of the cavity. These details are beyond the scope of our model.

The width of the shadows in the outer disk is dominated by the scale height of the inner disk, as it covers a wider solid angle of the star. A more detailed study could lead to a better constraint on the aspect ratio and flaring of the inner disk.

\section{DISCUSSION}

Warped disks are found in varied astrophysical contexts. Galactic warps may be due to a misalignment between a galaxy's angular momentum and its surrounding dark matter halo (Binney 1992) or by tidal encounters with nearby galaxies (Hunter \& Toomre 1969). Christiaens et al. (2014) propose that the two-armed spirals in the outer disk of HD 142527 might be indicative of a recent close stellar encounter (see also Quillen et al.2005). Although a flyby could also explain the tilt between the inner and outer disks, no partner for such a stellar encounter has been identified.

In the prototypical T-Tauri disk TW Hya, (Rosenfeld et al. 2012) proposed a warp to understand the sinusoidal $(m=1)$ azimuthal modulation seen in HST images, as well as features of the $\mathrm{CO}$ gas kinematics of the inner most regions. They found that a standard Keplerian disk model was unable to account for the CO line wings and spatially resolved emission near the central star, and explored 3 possible interpretations to account for the observed kinematics: 1) scaling up the temperature by a factor of three inside the cavity; 2) allowing super-Keplerian tangential velocities near the star, and 3) invoking a warped disk model in which the line-of-sight disk inclination increases towards the star. 
Dynamical interaction between circumstellar disks and (proto)planets or sub-stellar companions may lead to warps (Mouillet et al. 1997). In the $\beta$ Pictoris debris disk, the inner disk warp may have been dynamically induced by $\beta$ Pic b (Dawson et al.2011), whose orbit is found to be aligned with the inclined warped component (Lagrange et al.2012; Chauvin et al. 2012).

A warped inner disk in HD 142527 bears consequences on the physical conditions in the outer disk. The shadowed regions in the outer disk behind and along the inner disk midplane can be diagnosed in terms of temperature decrements in sub-mm continuum imaging (Casassus 2015b). There are other interesting consequences on the dynamics of the system. A question arises as to the origin of this warped disk, which is probably driven by the low-mass companion (Biller et al. 2012, Rodigas et al. 2014; Close et al. 2014), although it could also be linked with the disk-envelope interaction leading to the formation of the binary in the class I stage. Since there is no apparent displacement of the shadows by comparing the NICI images, acquired in June 2011, with the NACO PDI from July 2012, we can exclude the possibility that the inner tilt is precessing at a rate faster than $\sim 10 \mathrm{deg} \mathrm{yr}^{-1}$. Like- wise, we can discard shadows cast by compact concentrations of material in the very closest vicinity of the star.

The detailed scattered light images of HD 142527 available in the literature, coupled with state-of-the-art radiative transfer tools, have allowed us to conclude on the threedimensional structure of the system by levering on the inner disk shadows. The inner regions turn out to be warped, such that its inclination relative to the outer disk is $70 \pm 5 \mathrm{deg}$, and its PA is $-8 \pm 5 \mathrm{deg}$. This finding poses a challenge to understand the dynamics of the HD 142527 system, and is an invitation to interpret scattered light images of gapped protoplanetary disks from the perspective of inner warp shadows.

We thank Henning Avenhaus for providing comparison images in FITS format, and Jorge Cuadra for useful discussions. SM, SP and SC acknowledge support from the Millennium Science Initiative (Chilean Ministry of Economy), through grant "Nucleus P10-022-F". SM acknowledges CONICYTPCHA/MagisterNacional/2014 - folio 22140628. SP and SC acknowledge financial support from FONDECYT grants 3140601 and 1130949 , respectively.

\section{REFERENCES}

Avenhaus, H., Quanz, S. P., Schmid, H. M., Meyer, M. R., Garufi, A., Wolf, S., \& Dominik, C. 2014, ApJ, 781, 87

Biller, B., et al. 2012, ApJ, 753, L38

Binney, J. 1992, ARA\&A, 30, 51

Birnstiel, T., Dullemond, C. P. \& Pinilla, P. 2013, A\&A, 550, L8

Bohren, C. F., \& Huffman, D. 1983, Absorption and scattering of light by small particles, Wiley science paperback series (Wiley)

Canovas, H., Ménard, F., Hales, A., Jordán, A., Schreiber, M. R., Casassus, S., Gledhill, T. M., \& Pinte, C. 2013, A\&A, 556, A123

Casassus, S., Perez, S. M., van der Plas, G., Dent, W. R. F., Hales, A., \& Ménard, F. 2013a, NRAO workshop, "Transformational Science with ALMA: From Dust to Rocks to Planets"

Casassus, S., Perez M., S., Jordán, A., Ménard, F., Cuadra, J., Schreiber, M. R., Hales, A. S., \& Ercolano, B. 2012, ApJ, 754, L31

Casassus, S., et al. 2013b, Nature, 493, 191

Casassus, S. e. a. 2015a, in prep

-. 2015b, in prep

Castelli, F., \& Kurucz, R. L. 2003, in IAU Symposium, Vol. 210, Modelling of Stellar Atmospheres, ed. N. Piskunov, W. W. Weiss, \& D. F. Gray, 20P-+

Chauvin, G., et al. 2012, A\&A, 542, A41

Christiaens, V., Casassus, S., Perez, S., van der Plas, G., \& Ménard, F. 2014, submitted to ApJ

Close, L. M., et al. 2014, ApJ, 781, L30

Dawson, R. I., Murray-Clay, R. A., \& Fabrycky, D. C. 2011, ApJ, 743, L17

Dullemond, C., Juhasz, A., Pohl, A., Sereshti, F., Shetty, R., Peters, T., Commercon, B., \& Flock, M. 2014, RADMC3D v0.38

http://www.ita.uni-heidelberg.de/ dullemond/software/radmc-3d/
Fujiwara, H., et al. 2006, ApJ, 644, L133

Fukagawa, M., Tamura, M., Itoh, Y., Kudo, T., Imaeda, Y., Oasa, Y.,

Hayashi, S. S., \& Hayashi, M. 2006, ApJ, 636, L153

Fukagawa, M., et al. 2013, PASJ, 65, L14

Garcia Lopez, R., Natta, A., Testi, L., \& Habart, E. 2006, A\&A, 459, 837

Henning, T., \& Mutschke, H. 1997, A\&A, 327, 743

Hunter, C., \& Toomre, A. 1969, ApJ, 155, 747

Lagrange, A.-M., et al. 2012, A\&A, 542, A40

Li, A., \& Greenberg, J. M. 1997, A\&A, 323, 566

Lubow, S. H., \& D’Angelo, G. 2006, ApJ, 641, 526

Mouillet, D., Larwood, J. D., Papaloizou, J. C. B., \& Lagrange, A. M. 1997, MNRAS, 292, 896

Mulders, G. D., Paardekooper, S.-J., Panić, O., Dominik, C., van Boekel, R., \& Ratzka, T. 2013, A\&A, 557, A68

Ohashi, N. 2008, Ap\&SS, 313, 101

Perez, S., et al. 2014, ArXiv e-prints

Pinilla, P., Birnstiel, T., Ricci, L., Dullemond, C. P., Uribe, A. L., Testi, L., \& Natta, A. 2012, A\&A, 538, A114

Pontoppidan, K. M., Blake, G. A., \& Smette, A. 2011, ApJ, 733, 84

Quillen, A. C., Varnière, P., Minchev, I., \& Frank, A. 2005, AJ, 129, 2481

Rameau, J., Chauvin, G., Lagrange, A.-M., Thébault, P., Milli, J., Girard,

J. H., \& Bonnefoy, M. 2012, A\&A, 546, A24

Rodigas, T. J., Follette, K. B., Weinberger, A., Close, L., \& Hines, D. C. 2014, ApJ, 791, L37

Rosenfeld, K. A., et al. 2012, ApJ, 757, 129

van Boekel, R., et al. 2004, Nature, 432, 479

Verhoeff, A. P., et al. 2011, A\&A, 528, A91+ 